\title{
Evaluation of Traditional Chinese Medicine Continuous Nursing Effect on Functional Improvement of Elderly Stroke Patients
}

\author{
Y. L. LUO, Y. JIANG ${ }^{1}$, X. L. LUO ${ }^{2}$, X. Q. ZHONG* AND Z. R. ZHANG
}

Nursing Department, 'The Second Affiliated Hospital, Chongqing Three Gorges Medical College, Chongqing 404120,

${ }^{2}$ Cardiology, Daping Hospital Affiliated to Army Medical University, Chongqing 400042, China

Luo et al.: Traditional Chinese Medicine Continuous Nursing Effect on Elderly Stroke Patients

To evaluate the effect of traditional Chinese medicine continuous nursing on the functional improvement of elderly stroke patients. A total of 85 cases of elderly stroke patients admitted to the department of neurology of our hospital from January 2019 to January 2020 were selected as the research objects. According to the random number table method, the selected cases were divided into two groups, the control group (42 cases) received routine discharge nursing and the study group (43 cases) received traditional Chinese medicine continuous nursing on the basis of the control group. The cognitive function, neurological function, social function and motor function were compared between the two groups. Compared with those before intervention, the scores of Montreal cognitive assessment scale in the two groups were significantly increased after the intervention $(p<0.05)$ and Montreal cognitive assessment scale score of the study group was higher than that of the control group $(p<0.05)$. Compared with those before the intervention, the National Institutes of Health Stroke Scale scores of the two groups were significantly decreased after the intervention $(p<0.05)$ and the National Institutes of Health Stroke Scale score of the study group was lower than that of the control group $(p<0.05)$. Compared with those before the intervention, the Social Functional Rafing Scale scores of the two groups were significantly decreased after the intervention $(\mathbf{p}<\mathbf{0 . 0 5})$ and the Social Functional Rafing Scale score of the study group was lower than that of the control group $(\mathbf{p}<\mathbf{0 . 0 5})$. Compared with those before the intervention, the scores of Fugl-Meyer assessment (upper limb) and Fugl-Meyer assessment (lower limb) of the two groups were significantly increased after the intervention $(\mathbf{p}<0.05)$ and the scores of Fugl-Meyer assessment (upper limb) and FuglMeyer assessment (lower limb) of the study group were higher than those of the control group $(p<0.05)$. Traditional Chinese medicine continuous nursing has positive effects on improving the cognitive function, neural function, social function, motor function of elderly patients with stroke.

Key words: Traditional Chinese medicine continuous nursing, elderly stroke patients, functional improvement

Stroke is a common clinical cardiovascular and cerebrovascular disease with higher incidence among the elderly ${ }^{[1]}$. The survey shows that even after scientific treatment, most patients still suffer from varying degrees of dysfunction, such as cognitive, neurological and motor dysfunction, which seriously affects their health ${ }^{[2]}$. Therefore, rehabilitation intervention in addition to scientific treatment is quite necessary to reduce the disability rate. Most discharged patients who receive rehabilitation at home lack professional home based rehabilitation guidance and their health and safety issues during their stay at home are not properly resolved ${ }^{[3]}$. Traditional Chinese medicine (TCM) continuous nursing can guarantee nursing effect after discharge from hospital and provide targeted rehabilitation and nursing measures based on the patient's actual condition and relevant theories of TCM to reduce complications and promote recovery of

*Address for correspondence

E-mail: zhongxing425@126.com 
the damaged body function ${ }^{[4]}$. Based on this, the study implemented TCM continuous nursing for elderly stroke patients, with the intervention effect reported as follows.

\section{MATERIALS AND METHODS}

\section{General information:}

85 elderly stroke patients admitted to the Department of Neurology in our hospital from January 2019 to January 2020 were selected as the study subjects. The selected cases were divided into 2 groups according to random number table method. The control group had 42 cases, including 24 males and 18 females, aged $80-90 \mathrm{y}$, the patients had an average age of $(85.45 \pm 2.23)$ y. The study group had 43 patients, including 27 males and 16 females. Aged 81-90 y, the patients had an average age of $(85.51 \pm 2.25) \mathrm{y}$. In comparison of general data of the two groups, the difference was not statistically significant $(p>0.05)$, showing comparability.

\section{Ethical procedures:}

The medical ethics committee received the relevant materials of the applicant, including the ethics application form, the trial plan, the case report form and the informed consent of the subject.

The medical ethics committee conducted a preliminary review of the applicant's data and for those who met the requirements, submitted the protocol to the subjects.

Record and save test data until 3 y after the end of the test.

\section{Inclusion and exclusion criteria:}

Inclusion criteria-All meet the diagnostic criteria for stroke ${ }^{[5]}$; the patients have initial onset and are in the recovery period; age $\geq 80$ y old; patients have basic language communication ability, understanding ability and clear awareness; medical records are complete; patients and their families are informed and agree to participate in this study.

Exclusion criteria-Those suffering from malignant tumor; those suffering from malignant hypertension; those suffering from hearing impairment and aphasia; those with sudden deterioration of condition; those suffering from mental illness or dementia; there is (previous) alcohol/drug dependence.

\section{Nursing methods:}

The control group received routine nursing after discharge and was followed up by telephone once a week. Intervention period: 6 mo.

The study group received TCM continuous nursing on the basis of the control group. Intervention period: 6 mo. Specific contents are as follows:

A multidisciplinary medical intervention group was established. The members include rehabilitation therapists, nutritionists and psychologists, who work in the hospital with more than $5 \mathrm{y}$ of relevant work experience to be responsible for nursing in the corresponding professional field. There are also 5 nurses who work in the hospital with more than $3 \mathrm{y}$ of relevant work experience to be responsible for disease assessment and follow-up outside the hospital. A health file was established for each patient, including basic patient information (such as name, age, gender, etc.) and medical record information (such as treatment during hospitalization, dysfunction, etc.). The follow-up content and time were agreed before discharge from the hospital. The follow-up visit should be noted by phone $1 \mathrm{~d}$ in advance. After discharge, a nurse was responsible for telephone follow-up, once a week, while doctors and nurses were responsible for on-site follow-up, once a month. The team members cooperate with each other and collaborate in the formulation of a TCM continuous nursing plan that meets the patient's condition and nursing needs before the patient discharge from the hospital.

Health education with TCM characteristics; Refer to the "Chinese Guidelines for Cerebrovascular Disease Prevention and Treatment", "Nursing of Chinese Medicine", prepare "Stroke Prevention Knowledge Manual" covering diet, daily life, sports, etc., and distribute it to each patient, which can be accompanied by graphic presentations, video playback, etc.

Differentiation catering: Wind phlegm and blood stasis type-the main symptoms include hemiplegia, askew tongue, slurred speech, difficulty in articulation and loss of sensation. The second symptom was dizziness, phlegm and viscous, dim tongue, thin white tongue coating, slippery pulse. It is advisable to eat foods that can expel wind and remove blood stasis, clear heat and eliminate phlegm, like coix seed, radish, loquat, white fungus, etc., and avoid greasy and cold foods. The recommended dietary therapies include Chinese yam and sugar cane porridge; Pseudoginseng, Jujube and Crucian Carp soup; Salvia 
green tea and so on. Qi deficiency and blood stasis type-The main symptoms include hemiplegia, askew tongue, slurred speech, difficulty in articulation and hypoesthesia. Secondary symptoms include pale complexion, shortness of breath, tired god spent force, spontaneous perspiration, dim tongue, white greasy tongue coating or indented tongue edge, heavy pulse. It is advisable to eat qi invigorating and blood activating foods, such as pseudoginseng, red dates, roses, astragalus, etc., and avoid greasy and cold foods. The recommended dietary therapies include compound astragalus porridge, red dates groundnuts porridge and boiled chicken eggs with Chuanxiong, etc. Liver and kidney deficiency type-The main symptoms include hemiplegia, askew tongue, slurred speech, difficulty in articulation and loss of sensation. Secondary symptoms include tinnitus, warm hands, feet and heart, dry throat and mouth, red and thin tongue, less coating and rapid pulse. It is advisable to eat foods that nourish liver and kidney, supplement body essence and marrow, such as walnuts, lilies, mulberries, lean meat, etc., avoid greasy foods and acrid, excitatory products. The recommended dietary therapies include lycium soup, Mulberry cream, etc.

Rehabilitation exercise; the exercise plan was adjusted according to the rehabilitation situation of each patient, while the patient's family provided assistance, supervision. The recommended sports are: simple rehabilitation exercises, including bridge style movement, finger exercises, leg lifting exercises, etc.; low-intensity rehabilitation exercises, including Tai Chi, eight trigrams boxing, etc.; those with good physical strength can jog, climb stairs, etc.; exercise frequency: $20 \sim 45 \mathrm{~min} / 1 \mathrm{time} / \mathrm{d}$. The exercise had family member's accompaniment. One should stop immediately if discomfort is felt during the exercise. Warm up is needed before exercise and buffer exercise is required after exercise. In addition, heart rate should be controlled during exercise, which should not exceed 170 beats/min.

Emotional care; Medicine and food: Mum medlar tea can promote Qi and relieve depression; Xiaoyao powder can regulate vital energy and resolve depression. Five element music method: indignant person should appreciate velar sound based music, listen to energetic music. The recommended music includes "Green Grass", "Green Leaves Greet the Wind", etc.; joyful person should appreciate dental sound based music, listen to cheerful and relaxed music. The recommended music includes "Autumn Moon Over Han Palace", "Jolly Meeting", etc.; thoughtful person should appreciate guttural sound based music and listen to quiet, solemn music. The recommended music includes "Moonlit Night on Autumn Lake", "Horse Orchid", etc.; worried person should appreciate dental consonant based music and listen to high pitched and majestic music. The recommended music includes "Song of Yang Guan", "Mountain and Flowing Water", etc.; horrified person should appreciate labial sound-based music and listen to soft and smooth music. The recommended music includes "Song at the Frontier", "Serenade", etc. The psychologist downloads the corresponding music according to the patient's emotional state evaluation results. One with mixture of multiple emotions can listen alternately, $30 \mathrm{~min} / \mathrm{d}$.

Daily life time table should be adjusted according to seasonal changes. It is better to go to bed early and get up early in spring, sleep late and get up early with appropriate noon break in summer, fall asleep and get up early with noon break in autumn, sleep early and get up late in winter. Sleeping time should be no later than the midnight hour (23 1 o'clock), noon break should be at noon (11 13 o'clock), early sleep time is 21 o'clock, late sleep time should be not later than 23:00, early wake up time should be 5 7 o'clock, late wake up time is 7 o'clock. Avoid staying up late.

Chinese medicine nursing, acupoint massage based on syndrome differentiation; For wind phlegm and blood stasis type: pat the back along the meridian, i.e. the bladder meridians on both sides of the spine, tap gently in even rhythm from bottom to top, 70 times/ $\mathrm{min}, 20 \mathrm{~min} / \mathrm{time}, 2$ times/d. For Qi deficiency and blood stasis type: choose Zusanli, Shenshu points, Fenglong points, etc., massage for $20 \mathrm{~min}$ before sleep every day, or give chiropractic therapy, lift the skin with both hands along the sides of the spine, push forward while pinching, start from the caudal sacrum to the end of the occipital part, circulate for 5 times. For liver and kidney deficiency type: select Yongquan point, Shenshu point, Ganshu point, etc., massage for 20 min before sleep every day. Foot bath once a day before bedtime, $15 \mathrm{~min} /$ time with water temperature of $40^{\circ}$, water level should be higher than ankle joint. Massage with hands repeatedly during soaking.

\section{Observation indicators:}

Comparison of cognitive function: Evaluation tool is Montreal cognitive assessment scale (MoCA $)^{[6]}$, including executive function, attention, orientation, 
etc. MoCA has a total score of $0 \sim 30$ points and those with high scores have good cognitive function. It is evaluated once before and after intervention.

Comparison of neurological function: Evaluation tool is National Institute of Health Stroke Scale (NIHSS $)^{[7]}$. NIHSS has a total score of $0 \sim 42$ points. Those with high scores have a high degree of neurological dysfunction. It is evaluated once before and after intervention.

Comparison of social function: Evaluation tool is Social Functional Rating Scale (SFRS) ${ }^{[8]}$. SFRS includes 20 items, each item has a rating standard of $0 \sim 7$ points and 8 levels and those with high scores have severely impaired social function. It is evaluated once before and after intervention.

Comparison of motor function: Evaluation tool is Fugl-Meyer Activity (FMA) ${ }^{[9]}$. FMA (upper extremity) includes 33 items; each item has a rating standard of $0 \sim 2$ points and 3 levels and those with high scores have a low degree of motor dysfunction. FMA (lower extremity) includes 17 items and each item has a rating standard of $0 \sim 2$ points and 3 grades. Those with high scores have low motor dysfunction. It is evaluated once before and after intervention.

\section{Statistical processing:}

The data obtained from this study were analyzed using statistical software statistical package for the social sciences (SPSS) 22.0. The measurement data meeting normal distribution are expressed as mean \pm standard deviation. The independent sample $t$ test was used for comparison among groups and paired sample t test was used for intra group comparison. The test level is $\alpha=0.05, p<0.05$ indicates statistically significant difference.

\section{RESULTS AND DISCUSSION}

Comparison of cognitive function; before intervention, there is no statistically significant difference in MoCA scores between the two groups $(\mathrm{p}>0.05)$. After intervention, MoCA scores of both groups are significantly higher $(\mathrm{p}<0.05)$. Compared with the control group, the study group has higher MoCA score after intervention $(\mathrm{p}<0.05)$, as shown in Table 1.

Comparison of neurological function; before intervention, there is no statistically significant difference in NIHSS scores between the two groups $(p>0.05)$. After intervention, the NIHSS scores of both groups are significantly reduced $(p<0.05)$. Compared with the control group, the study group has lower NIHSS score after intervention $(\mathrm{p}<0.05)$, as shown in Table 2 .

Comparison of social function; before intervention, there is no significant difference in SFRS scores between the two groups ( $p>0.05)$. After intervention, the SFRS scores of both groups are significantly reduced $(p<0.05)$. Compared with the control group, the study group has lower SFRS score after intervention $(\mathrm{p}<0.05)$, as shown in Table 3.

Comparison of motor function; before intervention, there is no significant difference in FMA (upper extremity) score and FMA (lower extremity) score between the two groups ( $p>0.05)$. After intervention, FMA (upper extremity) score and FMA (lower extremity) score are significantly increased in both groups ( $\mathrm{p}>0.05)$. Compared with the control group, the study group has higher FMA (upper extremity) score and FMA (lower extremity) score after intervention $(\mathrm{p}<0.05)$, as shown in Table 4.

TABLE 1: COMPARISON OF COGNITIVE FUNCTION ( $\overline{\mathbf{x}} \pm \mathbf{s}$, POINT)

\begin{tabular}{lccccc}
\hline Group & Number of cases & Before intervention & After intervention & t & p \\
\hline Control group & 42 & $18.24 \pm 4.34$ & $20.59 \pm 5.82$ & 2.098 & 0.039 \\
Study group & 43 & $18.16 \pm 4.28$ & $23.67 \pm 6.19$ & 4.801 & 0.000 \\
$\mathrm{t}$ & - & 0.086 & 2.362 & - & - \\
$\mathrm{p}$ & - & 0.932 & 0.021 & - & - \\
\hline
\end{tabular}

TABLE 2: COMPARISON OF NEUROLOGICAL FUNCTION ( $\overline{\mathbf{x}} \pm \mathbf{s}$, POINT)

\begin{tabular}{lccccc}
\hline Group & Number of cases & Before intervention & After intervention & t & P \\
\hline Control group & 42 & $13.94 \pm 2.38$ & $8.07 \pm 1.92$ & 12.441 & 0.000 \\
Study group & 43 & $14.03 \pm 2.42$ & $5.03 \pm 1.49$ & 20.767 & 0.000 \\
$\mathrm{t}$ & - & 0.173 & 8.166 & - & - \\
$\mathrm{p}$ & - & 0.863 & 0.000 & - & - \\
\hline
\end{tabular}


TABLE 3: COMPARISON OF SOCIAL FUNCTION ( $\overline{\mathbf{x}} \pm \mathbf{s}$, POINT)

\begin{tabular}{lccccc}
\hline Group & Number of cases & Before intervention & After intervention & t & P \\
\hline Control group & 42 & $100.42 \pm 10.16$ & $80.75 \pm 24.27$ & 4.845 & 0.000 \\
Study group & 43 & $101.04 \pm 10.23$ & $50.18 \pm 15.55$ & 17.918 & 0.000 \\
$\mathrm{t}$ & - & 0.280 & 6.931 & - & - \\
$\mathrm{p}$ & - & 0.780 & 0.000 & - & - \\
\hline
\end{tabular}

TABLE 4: COMPARISON OF MOTOR FUNCTION $(x \pm s$, POINT)

\begin{tabular}{|c|c|c|c|c|c|c|c|c|c|}
\hline \multirow[b]{2}{*}{ Group } & \multirow{2}{*}{$\begin{array}{c}\text { Number of } \\
\text { cases }\end{array}$} & \multicolumn{4}{|c|}{ FMA (upper extremity) } & \multicolumn{4}{|c|}{ FMA (lower extremity) } \\
\hline & & $\begin{array}{c}\text { Before } \\
\text { intervention }\end{array}$ & $\begin{array}{c}\text { After } \\
\text { intervention }\end{array}$ & $\mathrm{t}$ & $\mathrm{p}$ & $\begin{array}{c}\text { Before } \\
\text { intervention }\end{array}$ & $\begin{array}{c}\text { After } \\
\text { intervention }\end{array}$ & $\mathrm{t}$ & $\mathrm{p}$ \\
\hline $\begin{array}{l}\text { Control } \\
\text { group }\end{array}$ & 42 & $35.23 \pm 8.11$ & $44.89 \pm 7.23$ & 5.762 & 0.000 & $18.09 \pm 4.12$ & $23.14 \pm 3.45$ & 6.090 & 0.000 \\
\hline $\begin{array}{l}\text { Study } \\
\text { group }\end{array}$ & 43 & $34.95 \pm 8.21$ & $54.28 \pm 6.16$ & 12.350 & 0.000 & $17.95 \pm 4.03$ & $29.86 \pm 2.97$ & 15.601 & 0.000 \\
\hline $\mathrm{t}$ & - & 0.158 & 6.451 & - & - & 0.158 & 9.631 & - & - \\
\hline $\mathrm{p}$ & - & 0.875 & 0.000 & - & - & 0.875 & 0.000 & - & - \\
\hline
\end{tabular}

Cognitive impairment is a common complication of stroke, among which vascular cognitive impairment is more frequent, with an incidence of $48.4 \% \sim 61$ $\%$. It is mainly manifested as memory loss, impaired executive ability, etc., which seriously affects the patient's life and comprehensive recovery. Studies have pointed out that vascular derived risk factors are often easy to identify with possibility of prevention and treatment. Proper intervention can delay the disease progression and reduce the damage degree to prevent progression into dementia. In this study, appropriate TCM continuous nursing intervention was performed during the stroke recovery period. The results showed that the study group had higher MoCA score than the control group after intervention, indicating that TCM continuous nursing can improve cognitive function of elderly stroke patients, which is consistent with the research of $\mathrm{Lin} \mathrm{Biyu}^{[10]}$. The analysis believes that implementation of TCM continuous nursing can ensure that patients still receive complete care during their stay at home. The brain has strong plasticity and reconstruction capabilities $^{[11]}$. According to the principles of TCM syndrome differentiation and holistic nursing, targeted health education with TCM characteristics and differentiation catering measures can promote the recovery of patient's cognitive function and effectively improve its outcomes.

Neurological dysfunction is also a common complication of stroke ${ }^{[12]}$. In this study, TCM continuous nursing was given. Where, daily life care, acupuncture point massage, etc. will help to improve blood supply of brain tissue and effectively improve the function of brain tissue cells and nerve cells throughout the body. Foot bath can directly stimulate the acupuncture points of the feet, which facilitates recovery of autonomic nerves and regeneration of brain cells, thus significantly promoting recovery of neurological function ${ }^{[13]}$. The results of this study indicated that the study group had lower NIHSS score than the control group after intervention, suggesting that TCM continuous nursing helps promote the recovery of damaged neurological function in elderly stroke patients.

Post stroke function recovery is a long process, which depends on the patient's correct and effective rehabilitation training and the patient's perseverance ${ }^{[14]}$. The implementation of TCM continuous nursing in this study can further improve the family care system. Where, emotional care can improve patients emotional state, rehabilitation exercise can promote the recovery of patients limb functions, regular follow-up can improve patient compliance ${ }^{[15]}$. Adjustment of the rehabilitation program according to the follow-up results can guarantee the continuity, effectiveness and scientificity of patient rehabilitation during stay at home. Implementation of multiple measures and close cooperation between multiple disciplines strengthen the patient's confidence in rehabilitation, improve the patient's self-care ability and reduce patient dependence on others. Therefore, social function can be significantly improved. The results of this study showed that: the study group had lower 
SFRS score than the control group after intervention, indicating that TCM continuous nursing can improve the social function of elderly stroke patients.

After the central nervous system is damaged, its motor function will be inhibited in varying degrees, thereby leading to motor dysfunction ${ }^{[16]}$. By stimulating particular acupoints, acupoint massage can dredge the channels, harmonize qi and blood, smoothen joints, link the exterior and interior and nourish the body, thereby improving muscle blood supply, oxygen supply, alleviating limb movement disorders and promoting motor function recovery. Rehabilitation movement is based on the patient's local dysfunction and can reconstruct the damaged motor function. In addition, regular follow-up can play a certain role of encouragement and supervision, significantly improve the rehabilitation motivation and compliance of patients and grasp the best opportunity for rehabilitation, so that the motor function can be significantly improved. The results of this study showed that the study group had higher FMA (upper extremity) score and FMA (lower extremity) score than the control group after intervention, indicating that TCM continuous nursing can improve motor function of elderly stroke patients $^{[17-19]}$.

In summary, TCM continuous nursing has demonstrated positive effects in improving the cognitive function, neurological function, social function and motor function of elderly stroke patients. This study still has the following limitations: single case source and small number of included cases, which may lead to generalization of research results; the intervention time is short, but elderly stroke rehabilitation is often a gradual and relative long process, therefore, it is necessary to extend the follow-up time to verify the long term intervention effect of TCM nursing. The TCM continuous care in this study is still in the development stage, no unified standard has been formed and whether the intervention is comprehensive remains to be further studied.

\section{Acknowledgements:}

The research is supported by National Science for Youth Foundation in 2017 "Yin Yang 1 (YY1) improves the curative efficiency of stem cell transplantation in myocardial infarction via SENP1/ HIF-1 $\alpha$-mediated anti-apoptotic effect of stem cells" (No. 81600230).

\section{Conflicts of interest:}

The authors declared no conflict of interest.

\section{REFERENCES}

1. Liu Y, Jin XL, Zhang Y, Zhou CY, Zhang ZC, Wang JW, et al. Research progress of Chinese and western medicine on clinical epidemiological characteristics of high-risk population of stroke. Chin J Integr Med Cardio Cerebrovasc Dis 2019;17(8):1180-3.

2. Cai XJ, Chen Y. Current status of stroke prevalence, prevention and control in our country. Guangdong Med J 2019;40:212-5.

3. Gan XB, Wu DY, Wang DY, Chen E, Chen LC, Chen RT. Effect of early rehabilitation intervention on quality of life in patients with acute ischemic stroke. Chin J Gerontol 2020;40(9):19925 .

4. Rangel ES, Belasco AG, Diccini S. Qualidade de vida de pacientes com acidente vascular cerebral em reabilitacao. Acta Paul Enferm 2013;26(2):205-12.

5. Chinese Society of Neurology, Chinese Stroke Society. Chinese guidelines for diagnosis and treatment of acute ischemic stroke 2018. Chin J Neurol 2018;51(9):666-82.

6. Guo XH. Effect of targeted nursing on perioperative period of elderly patients with femoral neck fracture undergoing artificial femoral head replacement. Guide Chin Med 2021;19(1):193-4.

7. Dong FH, Hu SL, Yue HL, Dong WJ. Effect of nursing intervention on postoperative hip disorder in elderly patients with hip fracture. Chin J Joint Surg 2017;11(5):496-9.

8. Gong LC, Liu F. Research progress on nursing devices in elderly stroke patients with urinary incontinence. Chin J Mod Nurs 2019;25(13):1605-8.

9. Zhao DT, Liu YF, Peng WY, Jiang XJ, Xue PP, Feng SL. Potential mechanisms of atmospheric PM2.5 impacting on cardiovascular health. Chem Life 2020;40(8):1228-34.

10. Nasreddine ZS, Phillips NA, Bedirian V, Charbonneau $\mathrm{S}$, Whitehead V, Collin I, et al. The Montreal Cognitive Assessment, MoCA: a brief screening tool for mild cognitive impairment. J Am Geriatr Soc 2005;53(4):695-9.

11. Dunning K. National Institutes of Health Stroke Scale. In: Kreutzer JS, DeLuca J, Caplan B editors. Encyclopedia of Clinical Neuropsychology. 9th ed. New York, NY: Springer; 2011. p. 1714-5.

12. Ren XF. Clinical value of continuous clinical nursing pathway to rehabilitation of patient with ischemia stroke. Guide Chin Med 2020;18(24):171-2.

13. Fugl-Meyer AR, Jaasko L, Leyman I, Olsson S, Steglind S. The post-stroke hemiplegic patient. 1. a method for evaluation of physical performance. Scand J Rehabil Med 1975;7(1):1331.

14. Qin L, Huang JY, Liao BG, Mao ZF, Wei J. Study on clinical characters and image features in patients with vascular cognitive impairment after ischemic stroke. China J Mod Med 2008;18(16):2398-401.

15. Yu XY, Wen MY, Chen XY. Effects of aerobic exercise combined with resistance exercise on neurological function, limb function and cognitive function in patients with nondementia cognitive impairment after stroke. J Clin Nurs 2019;18(5):33-6.

16. Lin BY. Clinical effect of acupuncture combined with traditional Chinese medicine rehabilitation on stroke patients with cognitive dysfunction. Liaoning J Tradit Chin Med 2018;45(3):605-8. 
17. Sun XQ, Zhang YJ, Yu YL, Liao RY. The effect of TCM differentiation on nerve and motor function in patients with acute ischemic stroke. Guiding J Tradit Chin Med Pharmacol 2018;24(7):113-5.

18. Fu HX. Effects of family empowerment nursing on the quality of nursing and improvement of negative emotion and social function of stroke patients. Mod Med J 2019;47(6):707-11.

19. Zeng CC. Effect of massage combined with modern rehabilitation exercise therapy on spasticity and limb motor function of elderly stroke patients. Hunan J Traditi Chin Med 2020;36(6):65-71
This is an open access article distributed under the terms of the Creative Commons Attribution-NonCommercial-ShareAlike 3.0 License, which allows others to remix, tweak, and build upon the work non-commercially, as long as the author is credited and the new creations are licensed under the identical terms

This article was originally published in a special issue,

"Therapeutic Perspectives in Biomedical Research and Pharmaceutical Sciences and their Nursing Methods"

Indian J Pharm Sci 2021:83(4)Spl issue "158-164" 